\section{Research Square}

Preprints are preliminary reports that have not undergone peer review.

They should not be considered conclusive, used to inform clinical practice, or referenced by the media as validated information.

\title{
Plasma NFL Disrupt Cognitive Integrity Via Coupling the Interactions of Core Subsystem and Frontoparietal Network in Alzheimer's Disease
}

Weina Yao

Nanjing Drum Tower Hospital: Nanjing University Medical School Affiliated Nanjing Drum Tower Hospital

Xiao Zhang

Nanjing Drum Tower Hospital: Nanjing University Medical School Affiliated Nanjing Drum Tower Hospital

Hui Zhao

Nanjing Drum Tower Hospital: Nanjing University Medical School Affiliated Nanjing Drum Tower Hospital

Yun Xu

Nanjing Drum Tower Hospital: Nanjing University Medical School Affiliated Nanjing Drum Tower Hospital

Feng Bai ( $\sim$ baifeng@njglyy.com )

Nanjing University Medical School Affiliated Nanjing Drum Tower Hospital https://orcid.org/0000-0001-7938-4729

Research

Keywords: Plasma neurofilament light chain, Alzheimer's disease, Core subsystem, Frontoparietal network,Cognitive integrity

Posted Date: August 30th, 2021

DOI: https://doi.org/10.21203/rs.3.rs-845199/v1

License: @ (1) This work is licensed under a Creative Commons Attribution 4.0 International License. Read Full License 


\section{Abstract}

Backgroud: Plasma neurofilament light chain (NFL) is a potential biomarker for neurodegenerative diseases. Both NFL and the interactions of core subsystem and frontoparietal network (FPN) are associated with the cognitive integrity. The present study was to investigate the underlying mechanism and application of plasmaNFL couplingcore-FPN in Alzheimer's disease (AD).

Methods: A total of 224 AD-spectrum participants with complete resting-state fMRI, neuropsychological tests and plasma NFL at baseline ( $n=112)$ and after approximately 17 months follow up $(n=112)$. Brain networks construction of core subsystem and FPN wereperformed in these subjects. The follow-up data were used to test and verify these baseline characteristics. Furthermore, Receiver Operating Characteristic analysiswas used to explore the classification by the association of plasma NFL and core-FPN, and the mediation analysis were appliedto investigate the significance of plasma NFL coupling networks on cognitive impairments in these subjects.

Results: The discernment ofdisease-relatedinteractions of core subsystem and FPN maybe the neural network fundamentals of plasmaNFL regarding to cognitive decline in AD-spectrum patients. Furthermore, the clinical significance of plasma NFL coupling networks on AD identification and monitoring cognitive impairments were observed in these subjects.

Conclusions: The characteristic change of plasma NFL coupling networks could expect to be used as an potential indicator of future targeted therapies and prevention strategies in $\mathrm{AD}$ patients.

\section{Introduction}

Alzheimer's disease (AD) is a neurodegenerative disorder characterized by cognitive impairment and progressive loss in the activities of daily life, imposing a heavy burden on society and family [1]. The current understanding of abnormal brain networks mechanisms was visible change within several years before the clinical symptoms of dementia [2]. In particular, the blood-based biomarkers reflect the full spectrum of disease triggering and driving molecular mechanisms underlying pathophysiological $\operatorname{AD}[3,4]$. This presents a unique opportunity for investigating the association of neuroimaging and blood-based biomarkers, as a novel measure of neurodegeneration and cognitive impairment.

During the long preclinical stage of the disease, at the macro level, AD is characterized by the failure of several brain networks, which may reflect the loss of homeostasis [5]. The default mode network (DMN) is as an intrinsic functional network, has been divided into three subsystems: the core subsystem, the dorsal medial prefrontal cortex (DMPFC) subsystem and the medial temporal lobe (MTL) subsystem [6]. In details, the DMPFC subsystem, mainly comprising the DMPFC, temporo-parietal junction, lateral temporal cortex and temporal pole, has been implicated in the decisions about one's present situation or mentalstate [7]. The MTL subsystem includes the ventral medial prefrontal cortex (vMPFC), posterior inferior parietal lobule, retrosplenial cortex, parahippocampal cortex and hippocampal formation, which is associated with memory processes, such as episodic memory retrieval [7]. Importantly, converging evidences have revealed that the core subsystem is proposed to be the hub linking the other two subsystems together, as well as the functional connectivity of the core subsystem is dependent of the disease which including the anterior medial prefrontal cortex (amPFC) and the posterior cingulate cortex (PCC) [6, 8-10]. Furthermore, our previous study has demonstrated that the core subsystem-centered connection abnormalities were associated with the progress of cognitive impairments in AD-spectrum patients [8].

Not only interactions within the DMN but also associations between the DMN and other large-scale networks, in particular the frontoparietal network (FPN) [11]. From the perspective of $A D$ pathology, $A \beta$ and tau were mainly deposited in the DMN and FPN $[12,13]$. From the perspective of functional interaction, DMN and FPN are cooperative rather than competitive networks [14, 15], and the interactions are associated with mnemonic processing [11]. The FPN comprises the lateral prefrontal cortex, dorsal anterior cingulate cortex, precuneus, anterior inferior parietal lobule, and the inferior temporal cortex, appear to be involved in instantiating and flexibly modulating cognitive control [16]. Moreover, the previous study has revealed core subsystem coupling with FPN during cognitive activity [17]. However, it remains unclear how core subsystem and FPN cohesively reconfigure in AD-spectrum patients, and whether such reconfigurations reflect behavioral performance.

Plasma neurofilament light chain (NFL) as elastic assemblies that help maintain cell shape, and the plasma NFL increases are considered to reflect axonal injury [18]. In AD spectrum patients, plasma NFL was related to cognitive decline in all tested cognitive measures over time [19, 20]. As such, it has been proposed as a blood-biomarker for monitoring AD progression in clinical practice and treatment trials [21-23]. Specifically, the previous studies addressing associations between plasma NFL and imaging markers were mainly in brain structural change. For example, in cognitively impaired subjects, the coupling between plasma NFL and grey matter loss was pronounced, particularly within the temporal lobe, as well as plasma NFL with small foci of white matter atrophy in the anterior and posterior cingulate [20]. In addition, the associations were seen with lateral ventricles, hippocampal volume, and cortex thickness of $A D$ at baseline and with the longitudinal change for imaging measures [21]. Emerging evidence has shown that the functional network is more vulnerable and sensitive than the structural alteration in AD patients [24, 25]. However, the interactions between plasma NFL and brain functional networks, in particular coreFPN have not yet been explored in AD-spectrum patients.

To our knowledge, this was the first study to address whether plasma NFL was associated with functional brain network abnormalities in the progression of AD. We hypothesized that plasma NFL could affect the interactions of core subsystem and FPN, which leads to the aggravation of clinical symptoms of ADspectrum patients. Importantly, the increasing knowledge on interacting neuroimaging and blood-based biomarkers in individuals suffering from AD may hold promise for the development of future targeted therapies and prevention strategies.

\section{Material And Methods}




\section{Alzheimer's Disease Neuroimaging Initiative}

Data used in the preparation of this article were obtained from the Alzheimer's Disease Neuroimaging Initiative (ADNI) database ( http://adni.loni . usc.edu). The ADNI was launched in 2003 as a public-private partnership by the National Institute on Aging (NIA), the National Institute of Biomedical Imaging and Bioengineering (NIBIB), the Food and Drug Administration (FDA), private pharmaceutical companies, and nonprofit organizations, as a \$60 million, 5-year public-private partnership. The primary goal of ADNI has been to test whether serial MRI, positron emission tomography, other biological markers, and clinical and neuropsychological assessment can be combined to measure the progression of $\mathrm{MCl}$ and early AD. The initiative is headed by Michael W. Weiner,MD,VA Medical Center and University of California, San Francisco. ADNI is the result of efforts of many co-investigators from a broad range of academic institutions and private corporations, and was approved by the institutional review board at each site and was compliant with the Health Insurance Portability and accountability Act. Written consent was obtained from all participants.

\section{Participants Characteristics}

The study selected participants matched in gender, age and education level from the ADNI2 and ADNI-GO. In the present study, a total of 224 participants with complete resting-state functional magnetic resonance imaging, neuropsychological tests and plasma NFL at baseline and a mean follow-up period of 17 months were eligible for inclusion. In details, an imaging analysis approach was utilized to investigate disease-related differences in test data (baseline, $\mathrm{n}=$ 112 ) and validation data (follow up, $n=112$ ), including 29 cognitively normal (CN) subjects, 35 early mild cognitive impairment (EMCI), 30 late mild cognitive impairment ( $\mathrm{LMCl})$ and $18 \mathrm{AD}$ patients both in baseline and follow up, respectively.

Clinical categorize was performed by the the central of ADNI based on diagnostic procedures from the ADNI protocol. Neuropsychologic assessments were used in the study including general cognition and episodic memory. General cognition containing Mini Mental State Examination (MMSE), Montreal Cognitive Assessment (MoCA), Clinical Dementia Rating (CDR), Alzheimer's Disease Assessment Scale-Cognitive section (ADAS) and Functional activities questionary (FAQ), while Ray Auditory Verbal Learning Test (RAVLT) can be regarded as a marker of episodic memory. Briefly, ADNI required participants with CN to have MMSE scores of 24-30, CDR of 0 , and no subjective or informant-reported memory decline and normal performance. The MCl are defined as including MMSE scores of 24-30, CDR of 0.5 and objective memory loss on the education adjusted Wechsler Memory. According to the degree of memory impairment of Wechsler memory scale (WMS), $\mathrm{MCl}$ is divided into EMCl and LMCl. AD participants showed memory decline with MMSE scores of 19-24, CDR of 0.5-1.0. For a detailed overview of full inclusion, exclusion and diagnostic criteria described are available in the ADNI procedure manual (http://www.adni-info.org/).

\section{Plasma Nfl Markers}

All subjects in this study had plasma NFL both in baseline and follow-up. Plasma NFL concentrations were analyzed using an in-house immunoassay on the Single molecule array (Simoa) HD-1 Analyser (Quanterix) as previously described. ${ }^{26}$ Analytical sensitivity of the assay was $<1.0 \mathrm{pg} / \mathrm{mL}, \mathrm{which}$ used a combination of monoclonal antibodies and purified bovine NFL as a calibrator.

\section{Mri Data Acquisition}

All participants were scanned using a 3.0-Tesla Philips MRI scanner. Resting-state fMRI scans images were obtained with an echo-planar imaging sequence covering the entire brain with the following parameters: repetition time $(T R)=3000 \mathrm{~ms}$; echo time $(T E)=30 \mathrm{~ms}$; flip angle $=80^{\circ}$; acquisition matrix $=64 \times 64$; number slices $=48$; slice thickness $=3.3 \mathrm{~mm}$; spatial resolution $=3.31 \times 3.31 \times 3.31 \mathrm{~mm}^{3}$. All imaging data were downloaded in the ADNI cohort and a detailed description of the resting-state fMRI data acquisition protocols also can be found at ADNI website (http://www.adni-info.org). The whole pipeline of four main sectors of image processing (a flowchart see Fig. 1), and the followings were the details.

\section{Image Processing}

The resting-state fMRI data were preprocessed using the Data Processing Assistant for Resting-State fMRI v2.2 (DPARSF) and Resting-State fMRI Data Analysis Toolkit (REST, http://restfmri.net), which is based on Matlab platform (Matlab R2013b). The data preprocessing steps were as follows. The first ten volumes of each data was discarded to uniform the signal equilibrium and eliminate subject's inadaptation to the scanning noise. Then remaining volumes were corrected to compensate for differences in acquisition time between slices and realignment to rectify head motion between scanning. The acquired functional images normalized to the Montreal Neurological Institute (MNI) space, and resampled into $3 \mathrm{~mm} \times 3 \mathrm{~mm} \times 3 \mathrm{~mm}$. Gaussian kernel ( $6 \times 6 \times 6 \mathrm{~mm})$ was applied to smooth the data. Band-pass filtering $(0.01-0.08 \mathrm{~Hz})$ was performed to reduce low frequency drift and high frequency noise due to physiological respiration and cardiac noise.

\section{Brain Networks Construction And Analysis}

\section{Network interaction based on the functional connectivity of core subsystem}

Our previous study has shown that core-centered connection abnormalities inside DMN subsystems at an early stage of AD [8]. In this study, we focused on the interactions of core subsystem associated with the outside of DMN subsystems and their clinical significance. First, the "Image Calculator" of REST was used to calculate the regions of core subsystem. Second, core subsystem served as seed for the functional connectivity of a voxel-wise analysis was used to 
explore the interactions between brain networks. For each subject, an average time series for core subsystem was computed as the reference time course. Pearson cross-correlation analysis was then conducted between the average signal change in the core subsystem and the time series of whole-brain voxels. Third, Fisher's z-transform $(z=0.5 \times \ln (1+r) /(1-r))$ improved the normality of the correlation coefficients, and the individual maps of each network was constructed. Finally, a voxel-wise analysis of variance (ANOVA) was performed in the baseline four groups. The statistical thresholds were set at a corrected $p$ $<0.05$, determined by Monte Carlo simulation for multiple comparisons (Parameters: single voxel $p$ value $=0.05$, a minimum cluster size of 6156 mm ${ }^{3}$, FWHM $=6 \mathrm{~mm}$, with mask. See program AlphaSim by D. Ward, and http://afni.nimh.nih.gov/pub/dist/doc/ manual/AlphaSim.pdf).

\section{Association analysis between core-related networks and plasma NFL}

We were particularly interested in these regions which were associated with statistically significant interactions of core-related networks and plasma NFL. Therefore, the regions of aforementioned ANOVA was extracted from each baseline participant's image. And then, a voxel-level correlation analysis was performed between these regions and plasma NFL in baseline AD-spectrum subjects (i.e., EMCl, $\mathrm{LMCl}$ and $\mathrm{AD}$ ). To remove possible effects of age, gender and education on the results, these parameters were introduced as covariates. To further remove spurious correlations, only those correlation coefficients were retained which corresponding $p$ values were lower than a statistical threshold ( $p<0.05$, determined by Monte Carlo simulation for multiple comparisons).

\section{Significance of plasma NFL coupling networks on groups identification}

To further explore the classification by the association of core-related networks and plasma NFL, these regions identified via baseline ANOVA and association analysis was extracted as region of interest (ROI). To avoid circular analysis, the mean Z values of every ROI were then calculated in all follow-up groups, respectively. Finally, the ability of ROls to separate these four follow-up groups (i.e., CN, EMCI, LMCl and AD) was computed using Receiver Operating Characteristic (ROC). Area under the ROC curve (AUC) values were extracted; the values distinguishing these follow-up four groups were examined. The thresholds were set at a $p<0.05$.

\section{Significance of plasma NFL coupling networks on cognitive impairments}

To determine the hypothesis of whether these aforementioned ROls mediated the relationships between plasma NFL and cognition, the mediation analysis was further performed. The primary estimates of interest were the behavioral significance in the extracted ROls both in baseline and follow-up data, respectively. We computed the bias-corrected $95 \%$ confidence intervals $(\mathrm{Cl})$ for the size of the mediating effects with bootstrap method $(\mathrm{k}=1000$ samples). The significance of the indirect effects was confirmed when $95 \%$ Cls were not contain zero. All the data were processed with the PROCESS for the Statistical Statistical Package for Social Sciences (SPSS) framework.

\section{Statistical Analysis Involving Demographic And Neuropsychological Data}

For demographic and neuropsychological information, the Kruskal-Wallis tests were used for the variables (i.e., age, education and behavioral scales) and the dichotomous variables (i.e., gender) while post-hoc tests were also performed by Mann-Whiney U Tests. The significance level was set at $p<0.05$ by SPSS version 22.

\section{Results}

\section{Demographic and behavioral characteristics}

As shown in Table 1, there were no significant differences in the age, gender or education levels of the participants in the $\mathrm{CN}, \mathrm{EMCl}, \mathrm{LMCl}$ and $\mathrm{AD}$ group ( $p>$ 0.05). Compared to $C N$ group, AD-spectrum subjects showed deficits in general cognition (i.e., MMSE, MoCA, CDR, ADAS11/13 and FAQ) and episodic memory (i.e., RAVLT_immediate, RAVLT_learning and RAVLT_perc_forgetting) both at baseline and follow up ( $p<0.05$, details see Table 1 and Table 2$)$. Furthermore, similar to previous findings [8], there was a disease stage-dependent pattern of progressively worsening cognitive impairments and increasing plasma NFL level with development of AD. However, self comparison baseline and a mean follow-up period of 17 months (Table 3 and Fig. 2), we observed that the late stage of disease (i.e., $\mathrm{LMCl}$ and $\mathrm{AD}$ ) was predominately significant reductions in general cognition and increased NFL level, while the deterioration of episodic memory was relatively stable. It should be noted that $\mathrm{CN}$ and $\mathrm{EMCl}$ showed no significant differences both in general cognition, episodic memory and $\mathrm{NFL}$ level, respectively.

\section{Interaction Between Core Subsystem And Fpn}

A qualitative visual inspection of the ANOVA of core subsystem and other networks in baseline four groups, and the distributed brain networks were demonstrated across the majority of the clusters including frontal, parietal cortical regions and subcortical sites in these groups (Fig. 3). We further explore these aforementioned regions which were associated with statistically significant interactions of core-related networks and plasma NFL in baseline ADspectrum subjects. Notably, all five regions of plasma NFL coupling networks were mainly located in FPN (i.e., right superior/middle/inferior frontal gyrus and bilateral inferior parietal lobule/supramarginal gyrus) (Fig. 3 and Table 4), confirming the interaction between core subsystem and FPN maybe as neural network fundamentals of plasma NFL regarding to cognitive decline in AD-spectrum subjects. A corrected threshold by Monte Carlo simulation was used at $p$ $<0.05$.

\section{Significance Of Plasma Nfl Coupling Networks On Groups Identification}

Page $4 / 13$ 
We further performed a application based on the present baseline findings. To avoid circular analysis, the ability of five regions to separate the four follow-up groups was explored. ROC analysis provided information on five regions regarding the classification of these follow-up groups, respectively (Fig. 4): (i) Right middle/inferior frontal gyrus: AUC is 0.706 ( $p=0.0146,95 \%$ Confidence Interval is 0.556 to 0.857$)$ in distinguishing EMCl from AD; AUC is 0.713 ( $p=0.0143$,

$95 \%$ Confidence Interval is 0.558 to 0.868 ) in distinguishing LMCI from AD; (ii) Left inferior parietal lobule/supramarginal gyrus: AUC is 0.652 ( $p=0.0454,95 \%$ Confidence Interval is 0.508 to 0.796 ) in distinguishing $\mathrm{LMCl}$ from $\mathrm{CN}$ group.

\section{Significance Of Plasma Nfl Coupling Networks On Cognitive Impairments}

To further explore the behavioural significance of plasma NFL coupling networks, we presented the results of the mediation analyses with the function of the regions of the coupling networks as a mediator, plasma NFL was entered as a predictor, and neuropsychological performance was an outcome. (i) In the baseline AD-spectrum subjects, there was a strong effect of plasma NFL on general cognition and episodic memory by the functional role of right superior frontal gyrus, right middle/inferior frontal gyrus and right inferior frontal gyrus/thalamus, respectively $(p<0.05$, details see Fig. 5). (ii) In the follow-up ADspectrum subjects, three priori ROIs including right superior frontal gyrus, right middle/inferior frontal gyrus and left inferior parietal lobule/supramarginal gyrus were as mediators, and plasma NFL affected general cognition and episodic memory by these mediators $(p<0.05$, details see Fig. 5).

\section{Discussion}

The present study was to investigate the functional brain network abnormalities related to plasma biomarker and their predictive value of cognitive decline in AD-spectrum patients. Specifically, there were two main findings. First, a novel observation was the discernment of disease-related interactions of core subsystem and FPN in AD-spectrum patients. Second, clinical significance of plasma NFL coupling networks in AD-spectrum patients was associated with AD identification and monitoring cognitive impairments. Together these findings provided evidences suggesting that disruptive effects of plasma NFL on cognitive integrity may operate via coupling the interactions of core subsystem and FPN in the progression of AD.

\section{Disease-related interactions of core subsystem and FPN in AD-spectrum patients}

These regions interacting with core subsystem, including frontal and parietal cortex, which were associated with FPN in AD-spectrum subjects. In AD patients, extracellular plaques of $\beta$-amyloid $(A \beta)$ fibrils and intracellular entanglement of hyperphosphorylated tau protein are abnormally formed. There is a growing amount of evidence that the frontal and parietal sites have decreased functional connectivity with increased $A \beta$, while tau protein accumulation first occurred in the DMN [27-29]. Therefore, the pathologically characteristic lesions in the progression of AD may be the basis of interactions based on the brain distribution of the FPN and core subsystem. In addition, due to its central role in the integrated adjustment, the FPN is called the central control network or executive control network which contained with dorsal attention network (DAN) and salience network (SN) [30, 31]. The FPN involved high-level cognitive field, for instance responsible for information scheduling, participating in problem decision and other processing tasks in working memory, as well as it had the strongest connections over the whole brain at the different cognitive functions [32, 33]. When the brain was confronted with different cognitive demands, the FPN can preferentially couple with DMN, as well as the FPN and DMN coordinate the reconstruction of the entire network reconfigurations by played different roles [15, 34]. In this study, we used the participation coefficient based on the sub-networks partition of Yan et al [9]. As the hub of DMN, core subsystem regulates high-order cognitive function depending on top-down [8]. In particular, the present findings contributed toward the understanding of the interactions of core subsystem and FPN maybe jointly affect cognition and as an important factor for later developing AD. However, the underlying mechanism of these coupling relationships between networks deserves further study.

\section{Clinical application value of plasma NFL coupling networks in AD-spectrum patients}

Plasma NFL, as an easily obtained specimen, has emerged as a blood-based biomarker in neuropathological changes [35]. Although increased NFL is not only associated with $A D$ but also caused neuronal injury in the central nervous system with a nonspecific features of pathophysiological processes [36, 37], the existing evidence demonstrated that the elevated of NFL has been associated with cognitive decline in AD-spectrum patients [20, 21, 38, 39]. Interestingly, plasma NFL increases with cognitively impaired in response to amyloid-related neuronal injury in preclinical stages of $A D$, but is related to tau-mediated neurodegeneration in cognitively impaired patients [19]. Neurodegeneration and axonal injury can be observed throughout the procession of AD, but with the increase of age and the progression of the AD they become more prominent [40]. Therefore, this may explain why the increasing plasma NFL levels was not observed until the later stage of dementia, with a significant difference between the baseline stage and the follow-up period of AD in the present study.

It should be noted that plasma NFL levels is a more universal biomarker of neuronal degeneration, and can be detected in many neurodegenerative diseases, which cannot be used as a specific indicator of $A D[26,41,42]$. Recent studies have shown that the concentrations of plasma NFL is significantly increased in both the prodromal and dementia stages of the sporadic AD, in which they closely related to disease-related structural brain changes [21, 38]. Here, this study conducted a mediation analysis to further investigate the roles of plasma NFL coupling networks. We found the neuropsychological performances were mediated by these coupling regions in AD-spectrum patients, supporting that plasma NFL as a noninvasive biomarker for AD has a clinical application value to differentiate between groups and monitor disease progression. Therefore, we assumed that plasma NFL cannot be used as a single indicator to detect $A D$, but when combined with the interactions of core subsystem and FPN, plasma NFL coupling networks could be used as a co-indicator and provide significant discriminant features for the classification of patients with cognitive impairments.

\section{Limitations}


Several limitations should be acknowledged in this study. First, this study did not has CSF NFL level based on the invasive procedure. It would be useful to examine the topologically convergent and divergent between CSF NFL and plasma NFL coupling networks in future studies. Second, we focused on the interactions of core subsystem and FPN, the more investigation including other networks could obtain more valuable information on the role of NFL. Finally, this study performed on a small sample size, we look forward to further studies with larger sample sizes to validate these findings. Therefore, these data should be interpreted with caution.

\section{Conclusions}

In summery, the present study provided evidence for the interactions of core subsystem and FPN, which maybe as neural network fundamentals of plasma NFL regarding to cognitive decline in AD-spectrum patients. We further identified the significance of plasma NFL coupling networks on $A D$ identification and monitoring cognitive impairments. Therefore, the characteristic change of plasma NFL coupling networks could expect to be used as an potential indicator of application in the progression of $A D$.

\section{Abbreviations}

NFL: Plasma neurofilament light chain; FPN:frontoparietal network; AD:Alzheimer's disease; DMN:default mode network; DMPFC:dorsal medial prefrontal cortex; MTL:medial temporal lobe; vMPFC:ventral medial prefrontal cortex; amPFC:anterior medial prefrontal cortex; PCC:posterior cingulate cortex; ADNI:Alzheimer's Disease Neuroimaging Initiative;

\section{Declarations}

\section{Acknowledgments}

Data collection and sharing for this project was in part funded by the Alzheimer's Disease Neuroimaging Initiative (ADNI) (National Institutes of Health Grant U01 AG024904) and DOD ADNI (Department of Defense award number W81XWH-12-2-0012). ADNI is funded by the National Institute on Aging, the National Institute of Biomedical Imaging and Bioengineering, and through generous contributions from the following: AbbVie, Alzheimer's Association; Alzheimer's Drug Discovery Foundation; Araclon Biotech; BioClinica, Inc.; Biogen; BristolMyers Squibb Company; CereSpir, Inc.; Cogstate; Eisai Inc.; Elan Pharmaceuticals, Inc.; Eli Lilly and Company; Eurolmmun; F. Hoffmann-La Roche Ltd and its affiliated company Genentech, Inc.; Fujirebio; GE Healthcare; IXICO Ltd.; Janssen Alzheimer Immunotherapy Research \& Development, LLC.; Johnson \& Johnson Pharmaceutical Research \& Development LLC.; Lumosity; Lundbeck; Merck \& Co., Inc.;Meso Scale Diagnostics, LLC.; NeuroRx Research; Neurotrack Technologies; Novartis Pharmaceuticals Corporation; Pfizer Inc.; Piramal Imaging; Servier; Takeda Pharmaceutical Company; and Transition Therapeutics. The Canadian Institutes of Health Research is providing funds to support ADNI clinical sites in Canada. Private sector contributions are facilitated by the Foundation for the National Institutes of Health (www.fnih.org). The grantee organization is the Northern California Institute for Research and Education, and the study is coordinated by the Alzheimer's Therapeutic Research Institute at the University of Southern California. ADNI data are disseminated by the Laboratory for Neuro Imaging at the University of Southern California.

\section{Authors' contributions}

WY: data analysis and interpretation.XZ: literature research. FB: funding acquisition, study concepts and study design. WY, XZ, HZ, YX and FB: editing and approval of the manuscript.

Funding This work was supported partly by grants from the National Natural Science Foundation of China (No. 81822013; 82071186).

\section{Availability of data and materials}

Not applicable.

\section{Ethics approval and consent to participate}

Data used in the preparation of this article were obtained from the ADNI database (http://adni.loni.usc.edu). Written consent was obtained from all participants.

\section{Consent for publication}

Not applicable.

\section{Competing interests}

The authors declare no competing fnancial interests.

\section{References}

1. Leng F, Edison P. Neuroinflammation and Microglial Activation in Alzheimer Disease: Where Do We Go from Here? Nat Rev Neurol. 2021;17(3):157-172.

2. Chen Y, Dang M, Zhang Z. Brain mechanisms underlying neuropsychiatric symptoms in Alzheimer's disease: a systematic review of symptom-general and -specific lesion patterns. Mol Neurodegener. 2021;16(1):38. 
3. Hampel H, O'Bryant SE, Molinuevo JL, Zetterberg H, L Masters C, Lista S, et al. Blood-based biomarkers for Alzheimer disease: mapping the road to the clinic. Nat Rev Neurol. 2018;14(11):639-652.

4. Thijssen EH, La Joie R, Wolf A, Strom A, Wang P, laccarino L, et al. Diagnostic value of plasma phosphorylated tau181 in Alzheimer's disease and frontotemporal lobar degeneration. Nat Med. 2020;26(3):387-397.

5. Castrillo Jl, Oliver SG. Alzheimer's as a systems-level disease involving the interplay of multiple cellular networks. Methods Mol Biol. 2016;1303:3-48.

6. Andrews-Hanna JR. The brain's default network and its adaptive role in internal mentation. Neuroscientist. 2012;18(3):251-70.

7. Bathelt J, Geurts HM. Difference in default mode network subsystems in autism across childhood and adolescence. Autism. 2021;25(2):556-565.

8. Yao W, Chen H, Sheng X, Zhao H, Xu Y, Bai F. Core-centered connection abnormalities associating with pathological features mediates the progress of cognitive impairments in AD-spectrum patients.J Alzheimers Dis. 2021;82(4):1499-1511.

9. Chen X, Chen NX, Shen YQ, Li HX, Li L, Lu B, et al. The subsystem mechanism of default mode network underlying rumination: A reproducible neuroimaging study. Neuroimage. 2020;221:117-185.

10. Tozzia L, Zhang X, Chesnuta M, Holt-Gosselin B, Ramirez CA, Williams LM. Reduced functional connectivity of default mode network subsystems in depression: Meta-analytic evidence and relationship with trait rumination. Neuroimage Clin. 2021;30:102570.

11. Buuren M, Wagner IC, Fernández G. Functional network interactions at rest underlie individual differences in memory ability. Learn Mem. 2018;26(1):9-19.

12. Villeneuve S, Rabinovici GD, Cohn-Sheehy BI, Madison C, Ayakta N, Ghosh PM, et al. Existing pittsburgh compound-B positron emission tomography thresholds are too high: statistical and pathological evaluation. Brain. 2015;138(Pt 7):2020-33.

13. Kazim SF, Seo JH, Bianchi R, Larson CS, Sharma A, Wong RKS, et al. Neuronal Network Excitability in Alzheimer's Disease: The Puzzle of Similar versus Divergent Roles of Amyloid $\beta$ and Tau. eNeuro. 2021;8(2):ENEURO.0418-20.2020.

14. Andrews-Hanna JR, Smallwood J, Spreng RN. The default network and self-generated thought: component processes, dynamic control, and clinical relevance. Ann N Y Acad Sci. 2014;1316(1):29-52.

15. Zuo N, Yang Y, Liu Y, Li J, Jiang T. Core networks and their reconfiguration patterns across cognitive loads. Hum Brain Mapp. 2018;39(9):3546-3557.

16. Scott Marek, Nico U. F. Dosenbach. The frontoparietal network: function, electrophysiology, and importance of individual precision mapping. Dialogues Clin Neurosci. 2018; 20(2): 133-140.

17. Su C, Zhou H, Wang C, Geng F, Hu Y. Individualized video recommendation modulates functional connectivity between large scale networks. Hum Brain Mapp. 2021. doi: 10.1002/hbm.25616. Online ahead of print.

18. Pereira JB, Janelidze S, Stomrud E, Palmqvist S, van Westen D, Dage JL,et al. Plasma markers predict changes in amyloid, tau, atrophy and cognition in non-demented subjects. Brain. 2021;awab163. doi: 10.1093/brain/awab163. Online ahead of print.

19. Benedet AL, Leuzy A, Pascoal TA, Ashton NJ, Mathotaarachchi S, Savard M,et al. Stage-specific links between plasma neurofilament light and imaging biomarkers of Alzheimer's disease. Brain. 2020;143(12):3793-3804.

20. Mattsson N, Cullen NC, Andreasson U, Zetterberg H, Blennow K. Association Between Longitudinal Plasma Neurofilament Light and Neurodegeneration in Patients With Alzheimer Disease. JAMA Neurol. 2019;76(7):791-799.

21. Mattsson N, Andreasson U, Zetterberg H, Blennow K. Association of plasma neurofilament light with neurodegeneration in patients with Alzheimer disease. JAMA Neurol. 2017;74(5):557-66.

22. Forgrave LM, Ma M, Best JR, DeMarco ML. The diagnostic performance of neurofilament light chain in CSF and blood for Alzheimer's disease, frontotemporal dementia, and amyotrophic lateral sclerosis: A systematic review and meta-analysis. Alzheimers Dement (Amst). 2019; 11: $730-743$.

23. Moscoso A, Grothe MJ, Ashton NJ, Karikari TK, Rodríguez JL, Snellman A,et al. Longitudinal Associations of Blood Phosphorylated Tau181 and Neurofilament Light Chain With Neurodegeneration in Alzheimer Disease. JAMA Neurol. 2021; 78(4): 1-12.

24. Kucikova L, Goerdten J, Dounavi ME, Mak E, Su L, Waldman AD, et al. Resting-state brain connectivity in healthy young and middle-aged adults at risk of progressive Alzheimer's disease. Neurosci Biobehav Rev. 2021;129:142-153.

25. Márquez F, Yassa MA. Neuroimaging Biomarkers for Alzheimer's Disease. Mol Neurodegener. 2019;14(1):21.

26. Gisslen M, Price RW, Andreasson U, Norgren N, Nilsson S, Hagberg L, et al. Plasma concentration of the NFL is a biomarker of CNS injury in HIV infection: a cross-sectional study. EBioMedicine. 2016; 3: 135-40

27. Wang L, Brier MR, Snyder AZ, Thomas JB, Fagan AM, Xiong C, et al. Cerebrospinal fluid A 42 , phosphorylated Tau181, and resting-state functional connectivity. JAMA Neurol. 2013;70(10):1242-8.

28. Villeneuve S, Rabinovici GD, Cohn-Sheehy BI, Madison C, Ayakta N, Ghosh PM, et al. Existing pittsburgh compound-B positron emission tomography thresholds are too high: statistical and pathological evaluation. Brain. 2015;138(Pt 7):2020-33.

29. Brier MR, Gordon B, Friedrichsen K, McCarthy J, Stern A, Christensen J, et al. Tau and A $\beta$ imaging, CSF measures, and cognition in Alzheimer's disease. Sci Transl Med. 2016, 8(338):338-366.

30. Melrose RJ, Jimenez AM, Riskin-Jones H, Weissberger G, Veliz J, Hasratian AS, et al. Alterations to task positive and task negative networks during executive functioning in mild cognitive impairment. Neuroimage Clin. 2018;19:970-981.

31. Cai S, Peng Y, Chong T, Zhang Y, von Deneen KM, Huang L, et al. Differentiated Effective Connectivity Patterns of the Executive Control Network in Progressive MCI $\mathbb{A}$ A Potential Biomarker for Predicting AD. Curr Alzheimer Res. 2017;14(9):937-950.

32. Philippi CL, Pujara MS, Motzkin JC, Newman J, Kiehl KA, Koenigs M. Altered resting-state functional connectivity in cortical networks in psychopathy. Neurosci. 2015;35(15):6068-78

33. Petrides M. Lateral prefrontal cortex $\rrbracket$ architectonic and functional organization. Philos Trans R Soc Lond B Biol Sci. 2005; $360(1456) \varangle 781-95$ 
34. Spreng RN, Stevens W D, Chamberlain JP, Gilmore AW, Schacter DL. Default network activity, coupled with the frontoparietal control network, supports goal-directed cognition. Neuroimage. 2010;53(1):303-17.

35. Khalil M, Teunissen CE, Otto M, Piehl F, Sormani MP, Gattringer T,et al. Neurofilaments as biomarkers in neurological disorders. Nat Rev Neurol. 2018;14(10):577-589.

36. Bridel C, van Wieringen WN, Zetterberg H, Tijms BM, Teunissen CE, Alvarez-Cermeño JC, et al. Diagnostic value of cerebrospinal fluid neurofilament light protein in neurology: a systematic review and meta-analysis. JAMA Neurol. 2019;76(9),1035-1048.

37. Canto E, Barro C, Zhao C, Caillier SJ, Michalak Z, Bove R, et al. Association between serum neurofilament light chain levels and long-term disease course among patients with multiple sclerosis followed up for 12 years. JAMA Neurol. 2019;76(11):1359-1366.

38. Zetterberg H, Skillbäck T, Mattsson N, Trojanowski JQ, Portelius E, Shaw LM, et al. Association of cerebrospinal fluid neurofilament light concentration with Alzheimer disease progression. JAMA Neurol. 2017;74(1):557-566.

39. Lin YS, Lee WJ, Wang SJ, Fuh JL. Levels of plasma neurofilament light chain and cognitive function in patients with Alzheimer or Parkinson disease. Sci Rep. 2018;8(1):17368.

40. Jack Jr CR, Bennett DA, Blennow K, Carrillo MC, Dunn B, Haeberlein SB, et al. NIA-AA Research Framework: Toward a biological definition of Alzheimer's disease. Alzheimers Dement. 2018;14(4):535-562.

41. Sun Y, Tan L, Xu W, Wang ZT, Hu H, Li JQ, et al. Plasma neurofilament light and longitudinal progression of white matter hyperintensity in elderly persons without dementia. J Alzheimers Dis. 2020;75(3):729-737.

42. Rojas JC, Karydas A, Bang J, Tsai RM, Blennow K, Liman V, et al. Plasma neurofilament light chain predicts progression in progressive supranuclear palsy. Ann Clin TransI Neurol. 2016;3(3):216-225.

\section{Tables}

\begin{tabular}{|c|c|c|c|c|}
\hline Items & $\begin{array}{l}\mathrm{CN} \\
(n=29)\end{array}$ & $\begin{array}{l}\mathrm{EMCl} \\
(\mathrm{n}=35)\end{array}$ & $\begin{array}{l}\mathrm{LMCl} \\
(\mathrm{n}=30)\end{array}$ & $\begin{array}{l}A D \\
(n=18)\end{array}$ \\
\hline
\end{tabular}

\section{Demographics}

\begin{tabular}{|c|c|c|c|c|c|}
\hline Age (years) & $74.10 \pm 5.69$ & $70.96 \pm 7.21$ & $72.20 \pm 7.63$ & $73.61 \pm 8.06$ & 0.26 \\
\hline Education (years) & $16.45 \pm 2.37$ & $15.43 \pm 2.56$ & $16.73 \pm 2.33$ & $15.17 \pm 2.55$ & 0.065 \\
\hline Gender (male/female) & $15 / 14$ & $17 / 18$ & $18 / 12$ & $8 / 10$ & 0.722 \\
\hline \multicolumn{6}{|l|}{ General cognition } \\
\hline MMSE & $28.86 \pm 1.33$ & $27.97 \pm 1.84$ & $27.67 \pm 1.60$ & $22.33 \pm 2.4$ & $<0.001$ \\
\hline MoCA & $25.24 \pm 1.81$ & $23.80 \pm 3.06$ & $22.30 \pm 3.10$ & $16.83 \pm 5.19$ & $<0.001$ \\
\hline CDR & $0.07 \pm 0.18$ & $1.41 \pm 0.97$ & $1.70 \pm 0.97$ & $4.69 \pm 2.08$ & $<0.001$ \\
\hline ADAS11 & $6.10 \pm 2.54$ & $7.80 \pm 3.3$ & $11.13 \pm 4.73$ & $23.56 \pm 7.36$ & $<0.001$ \\
\hline ADAS13 & $9.90 \pm 3.51$ & $12.17 \pm 5.00$ & $17.57 \pm 7.05$ & $34.83 \pm 6.60$ & $<0.001$ \\
\hline FAQ & $0.03 \pm 0.19$ & $2.11 \pm 3.55$ & $4.87 \pm 5.11$ & $15.11 \pm 8.40$ & $<0.001$ \\
\hline \multicolumn{6}{|l|}{ Episodic Memory } \\
\hline RAVLT_immediate & $43.59 \pm 9.49$ & $37.83 \pm 10.80$ & $32.93 \pm 7.45$ & $22.06 \pm 6.16$ & $<0.001$ \\
\hline RAVLT_learning & $5.59 \pm 2.51$ & $5.60 \pm 2.67$ & $3.40 \pm 2.31$ & $1.00 \pm 1.64$ & $<0.001$ \\
\hline RAVLT_forgetting & $4.14 \pm 2.64$ & $4.94 \pm 1.97$ & $4.90 \pm 2.83$ & $4.11 \pm 1.08$ & 0.123 \\
\hline RAVLT_perc_forgetting & $40.74 \pm 27.87$ & $56.02 \pm 28.15$ & $64.58 \pm 30.30$ & $92.79 \pm 14.25$ & $<0.001$ \\
\hline \multicolumn{6}{|l|}{ Blood biomarker } \\
\hline Plasma NFL & $36.08 \pm 11.31$ & $33.07 \pm 13.85$ & $36.35 \pm 17.09$ & $45.46 \pm 18.25$ & 0.174 \\
\hline \multicolumn{6}{|c|}{$\begin{array}{l}\text { Note: Values were presented as the average } \pm \text { standard error (SE); } p \text {-value, because the data do not satisfy the normal distribution, the } p \text { value was obtained } k \\
\text { here due to the fact that the data were not normally distributed; *indicates a statistical difference between groups, } p<0.05 \text {. Abbreviations: CN, cognitively nor } \\
\text { cognitive impairment; AD, Alzheimer's disease; CDR, Clinical Dementia Rating; ADAS, Alzheimer's Disease Assessment Scale-Cognitive section; FAQ, Function } \\
\text { MoCA, Montreal Cognitive Assessment; RAVLT, Rey Auditory Verbal Learning Test; Plasma NFL, Plasma neurofilament light chain. }\end{array}$} \\
\hline
\end{tabular}




\begin{tabular}{|c|c|c|c|c|c|}
\hline Items & $\begin{array}{l}\mathrm{CN} \\
(n=29)\end{array}$ & $\begin{array}{l}\mathrm{EMCl} \\
(\mathrm{n}=35)\end{array}$ & $\begin{array}{l}\mathrm{LMCl} \\
(\mathrm{n}=30)\end{array}$ & $\begin{array}{l}A D \\
(n=18)\end{array}$ & $\begin{array}{l}p- \\
\text { Value }\end{array}$ \\
\hline \multicolumn{6}{|l|}{ General cognition } \\
\hline MMSE & $29.07 \pm 1.00$ & $28.14 \pm 1.73$ & $26.13 \pm 2.60$ & $18.78 \pm 4.15$ & $<0.001$ \\
\hline MoCA & $26.48 \pm 2.35$ & $23.37 \pm 3.48$ & $21.67 \pm 3.80$ & $14.00 \pm 5.03$ & $<0.001$ \\
\hline CDR & $0.12 \pm 0.26$ & $1.31 \pm 1.31$ & $2.47 \pm 1.74$ & $5.97 \pm 2.82$ & $<0.001$ \\
\hline ADAS11 & $5.45 \pm 2.56$ & $10.43 \pm 9.67$ & $13.03 \pm 7.09$ & $27.67 \pm 9.17$ & $<0.001$ \\
\hline ADAS13 & $7.90 \pm 3.53$ & $15.17 \pm 12.33$ & $19.33 \pm 9.33$ & $40.11 \pm 10.62$ & $<0.001$ \\
\hline FAQ & $0.28 \pm 0.75$ & $3.17 \pm 4.97$ & $5.80 \pm 6.92$ & $18.72 \pm 8.38$ & $<0.001$ \\
\hline \multicolumn{6}{|l|}{ Episodic Memory } \\
\hline RAVLT_immediate & $45.45 \pm 9.85$ & $37.77 \pm 11.99$ & $32.10 \pm 11.04$ & $19.78 \pm 6.93$ & $<0.001$ \\
\hline RAVLT_learning & $5.79 \pm 2.24$ & $5.17 \pm 2.94$ & $3.83 \pm 2.64$ & $1.50 \pm 1.42$ & $<0.001$ \\
\hline RAVLT_forgetting & $4.41 \pm 2.73$ & $5.03 \pm 2.44$ & $4.70 \pm 1.78$ & $4.39 \pm 1.58$ & 0.297 \\
\hline RAVLT_perc_forgetting & $41.09 \pm 27.31$ & $59.73 \pm 33.96$ & $68.41 \pm 30.68$ & $97.22 \pm 11.79$ & $<0.001$ \\
\hline \multicolumn{6}{|l|}{ Blood biomarker } \\
\hline Plasma NFL & $43.96 \pm 38.38$ & $37.48 \pm 29.04$ & $43.78 \pm 22.25$ & $56.56 \pm 22.97$ & $0.004^{*}$ \\
\hline \multicolumn{6}{|c|}{$\begin{array}{l}\text { Note: Values were presented as the average } \pm \text { standard error (SE); } p \text {-value, because the data do not satisfy the normal distribution, the } p \text { value was obtained } k \\
\text { here due to the fact that the data were not normally distributed; *indicates a statistical difference between groups, } p<0.05 \text {. Abbreviations: CN, cognitively nor } \\
\text { cognitive impairment; AD, Alzheimer's disease; CDR, Clinical Dementia Rating; ADAS, Alzheimer's Disease Assessment Scale-Cognitive section; FAQ, Function } \\
\text { MoCA, Montreal Cognitive Assessment; RAVLT, Rey Auditory Verbal Learning Test. }\end{array}$} \\
\hline
\end{tabular}

Table 3. Neuropsychological difference between baseline and follow up. 


\begin{tabular}{|c|c|c|c|c|}
\hline Items & $\triangle \mathrm{CN}$ & $\triangle \mathrm{EMCl}$ & $\triangle \mathrm{LMCl}$ & $\triangle \mathrm{AD}$ \\
\hline \multicolumn{5}{|l|}{ General cognition } \\
\hline MMSE & $-0.21 \pm 0.33$ & $-0.17 \pm 0.30$ & $26.13 \pm 2.60$ & $3.56 \pm c$ \\
\hline MoCA & $-1.24 \pm 0.47$ & $0.43 \pm 0.37$ & $0.63 \pm 0.50$ & $2.83 \pm c$ \\
\hline CDR & $-0.05 \pm 0.05$ & $0.100 \pm 0.14$ & $-0.77 \pm 0.22$ & $-1.28 \pm$ \\
\hline ADAS11 & $0.66 \pm 0.42$ & $-2.63 \pm 1.49$ & $-1.90 \pm 0.80$ & $-4.11 \pm$ \\
\hline ADAS13 & $2.00 \pm 0.52$ & $-3.00 \pm 1.67$ & $-1.77 \pm 0.89$ & $-5.28 \pm$ \\
\hline FAQ & $-0.24 \pm 0.14$ & $-1.06 \pm 0.56$ & $-0.93 \pm 0.79$ & $-3.61 \pm$ \\
\hline \multicolumn{5}{|l|}{ Episodic Memory } \\
\hline RAVLT_immediate & $-1.86 \pm 7.64$ & $0.06 \pm 1.30$ & $0.83 \pm 1.48$ & $2.28 \pm c$ \\
\hline RAVLT_learning & $-0.21 \pm 0.47$ & $0.43 \pm 0.42$ & $-0.43 \pm 0.35$ & $-0.50 \pm$ \\
\hline RAVLT_forgetting & $-0.28 \pm 0.55$ & $-0.09 \pm 0.34$ & $0.20 \pm 3.00$ & $-0.28 \pm$ \\
\hline RAVLT_perc_forgetting & $-0.46 \pm 4.52$ & $-3.71 \pm 3.47$ & $-3.83 \pm 6.34$ & $-4.43 \pm$ \\
\hline \multicolumn{5}{|l|}{ Blood biomarker } \\
\hline Plasma NFL & $-7.89 \pm 6.29$ & $-4.41 \pm 4.04$ & $-7.43 \pm 2.93$ & $-11.1 \pm$ \\
\hline
\end{tabular}

Table 4. The regions associated with plasma NFL coupling networks

\begin{tabular}{|c|c|c|c|c|}
\hline Brain region & BA & $\begin{array}{l}\text { Peak MNI } \\
\text { Coordiates } x, y, z(m m)\end{array}$ & Peak intensity & $\begin{array}{l}\text { Cluster } \\
\text { size }\end{array}$ \\
\hline R Superior Frontal Gyrus & $6 / 8$ & $6,24,54$ & 0.333 & 6318 \\
\hline R Middle/Inferior Frontal Gyrus & $10 / 11 / 47$ & 5439,0 & 0.464 & 13446 \\
\hline R Inferior Frontal Gyrus/Thalamus & $44 / 9$ & $3,-21,6$ & 0.429 & 22815 \\
\hline L Inferior Parietal Lobule/Supramarginal Gyrus & 40 & $-39,-51,42$ & 0.36 & 6183 \\
\hline R Inferior Parietal Lobule/Supramarginal Gyrus & 40 & $42,-48,33$ & 0.445 & 17064 \\
\hline
\end{tabular}

\section{Figures}



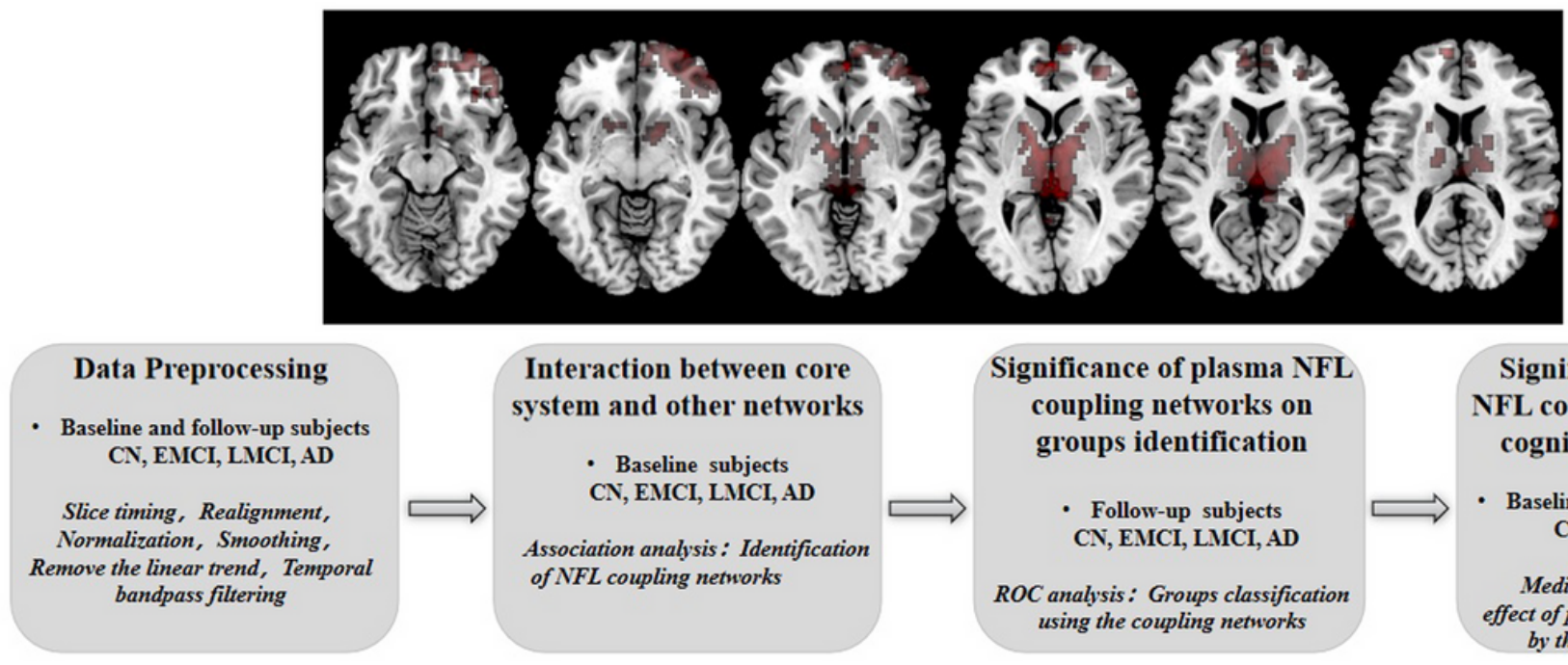

Significance of plasma NFL coupling networks on groups identification

- Follow-up subjects CN, EMCI, LMCI, AD

ROC analysis: Groups classification using the coupling networks
Significance of plasma NFL coupling networks on cognitive imapirments

Baseline and follow-up subjects CN, EMCI, LMCI, AD

Mediation analysis: Mediated effect of plasma NFL on cognition by the coupling networks

Figure 1

A flowchart shows the whole pipeline of four main sectors of image processing, including (i) Data preprocessing, (ii) Association analysis: Identification of NFL coupling networks, (iii) ROC analysis: Groups classification using the coupling networks and (iv) Mediation analysis: Mediated effect of NFL on cognition by the coupling networks.

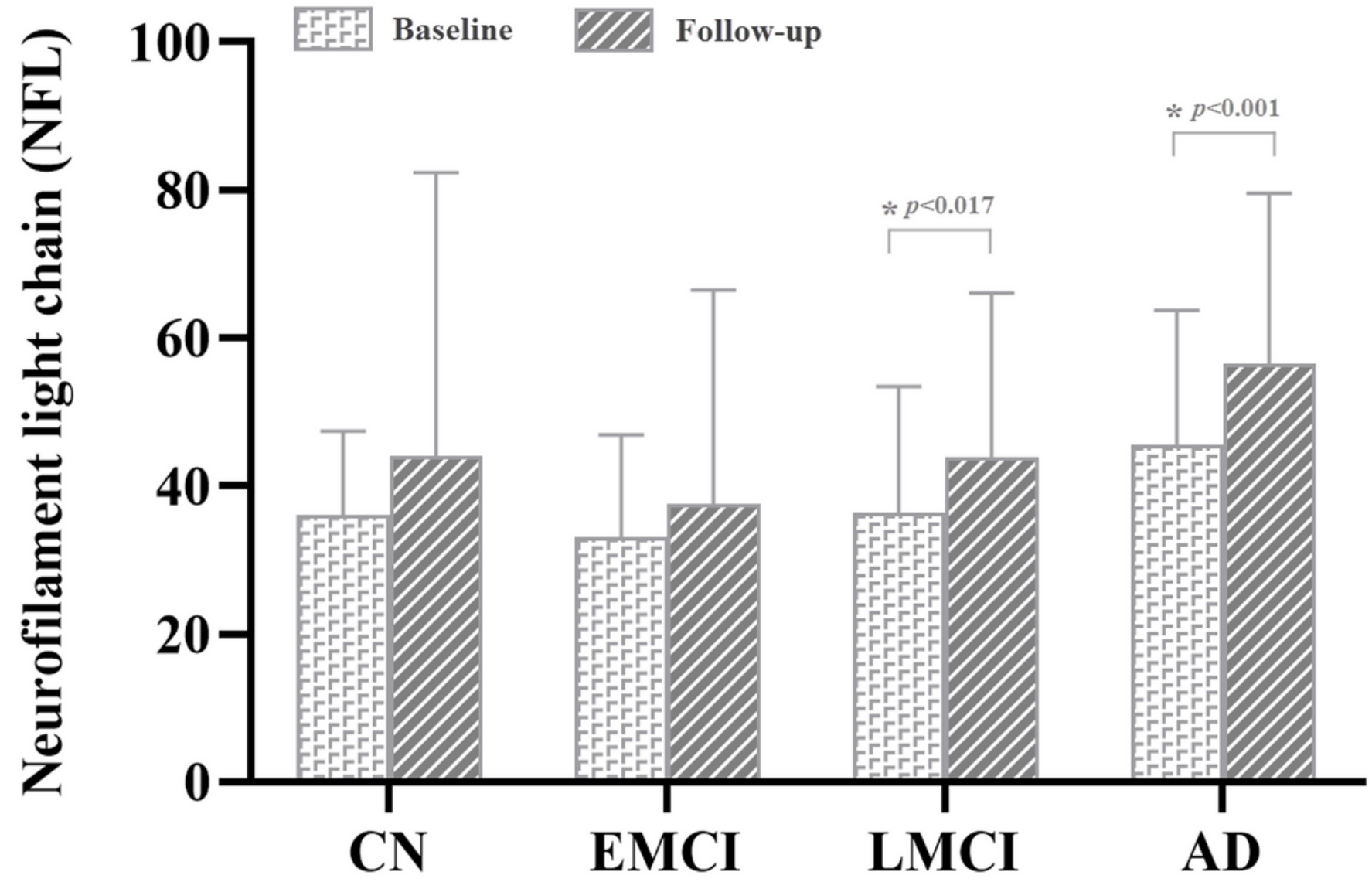

Figure 2

Compared to baseline, the late stage of disease (i.e., $\mathrm{LMCl}$ and $\mathrm{AD}$ ) was predominatelysignificant reductionsin increased $\mathrm{NFL}$ level(p< 0.05$)$, whileCN and $\mathrm{EMCl}$ showed no significant differences in NFL level at a mean follow-up period of 17 months, respectively. 
Interaction between core system and other networks

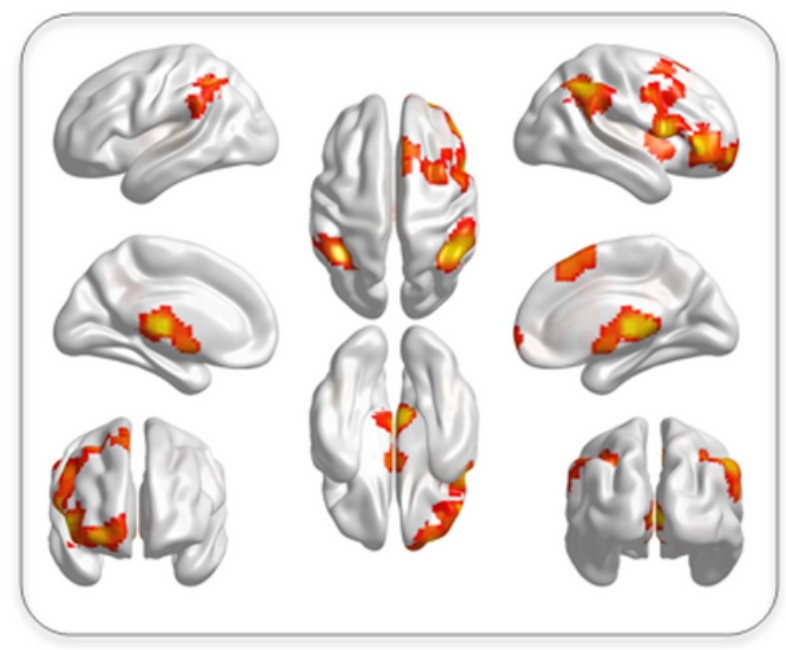

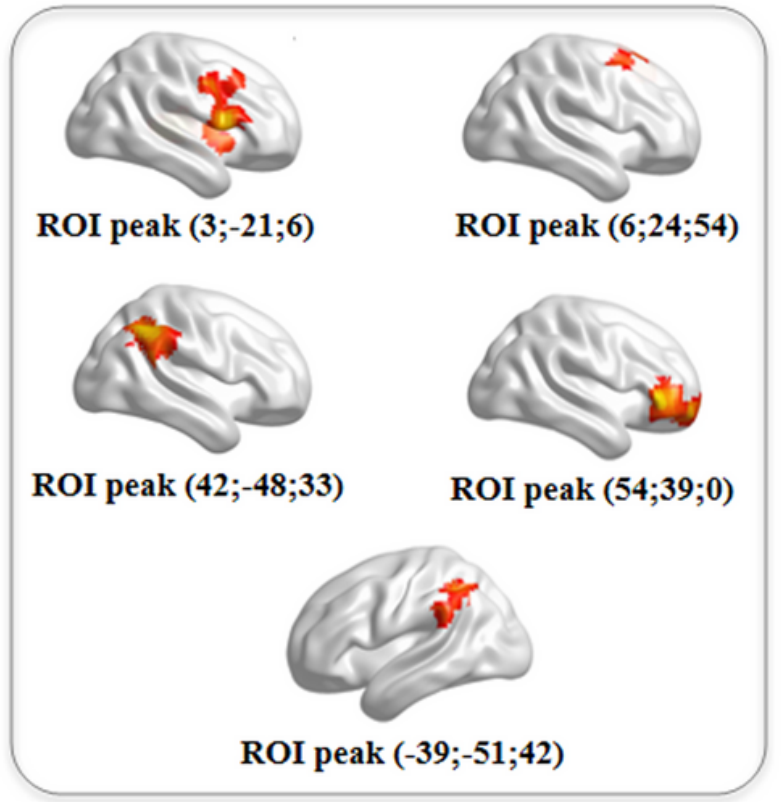

Figure 3

A qualitative visual inspection of the interactions between core subsystem and FPN in baseline four groups,and the distributed brain networks were demonstrated across the majority of the clusters including frontal, parietal cortical regions and subcortical sites in these groups ( $p<0.05$ corrected by Monte Carlo simulation).Notably, plasma NFLcoupling networkswere mainly located in the five regions of FPN, including right inferior frontal gyrus/thalamus(MNI: 3 , $-21,6)$, right superior frontal gyrus (MNI: 6,24, 54), rightinferior parietal lobule/supramarginal gyrus (MNl: $42,-48,33)$, rightmiddle/inferior frontal gyrus (MNI: $5439,0)$ and leftinferior parietal lobule/supramarginal gyrus(MNI: $-39,-51,42)(p<0.05$ corrected by Monte Carlo simulation).

\section{Significance of plasma NFL coupling networks on groups identification}
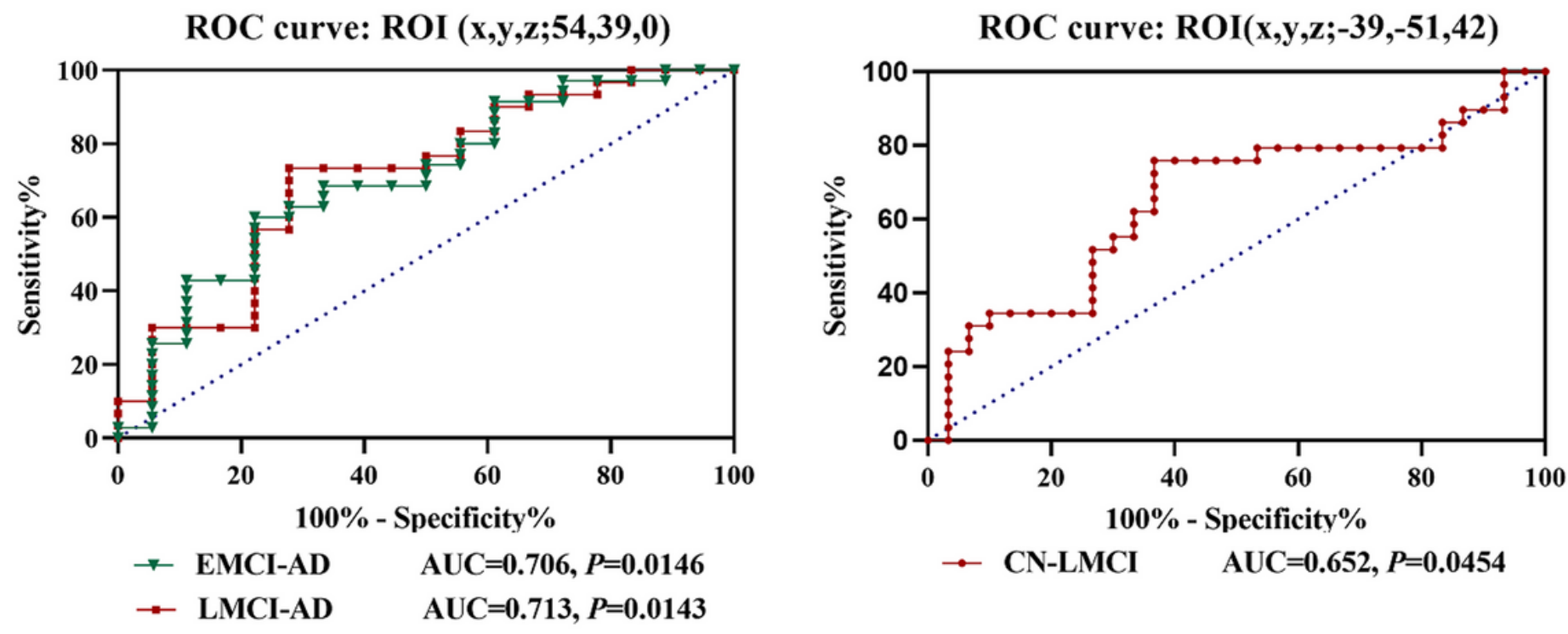

Figure 4

Mean Z values of every region associated with plasma NFL coupling networks regarding the classification of the follow-up groups, respectively. (i)Rightmiddle/inferior frontal gyrus(MNI: 5439,0$)$ :AUC is 0.706 ( $p=0.0146,95 \%$ Confidence Interval is 0.556 to 0.857 ) in distinguishing EMCI from AD; AUC is $0.713(p=0.0143,95 \%$ Confidence Interval is 0.558 to 0.868 ) in distinguishing LMCl from AD; (ii) Leftinferior parietal lobule/supramarginal gyrus (MNI: -39 , $-51,42)$ : AUC is 0.652 ( $p=0.0454,95 \%$ Confidence Interval is 0.508 to 0.796 ) in distinguishing LMCI from CN group. 


\section{Significance of plasma NFL coupling networks on cognitive impairments}

Baseline AD-spectrum patients
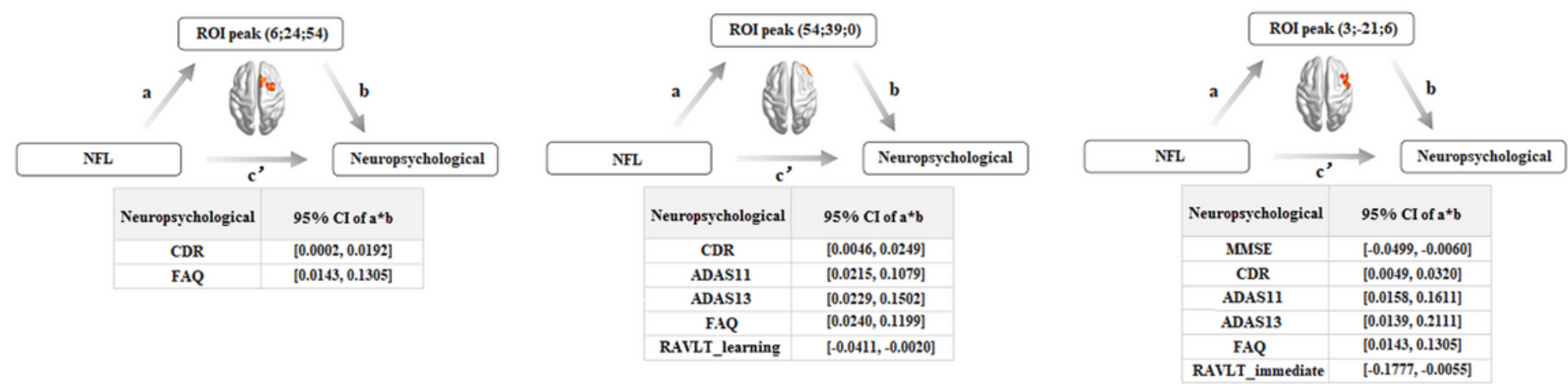

Follow-up AD-spectrum patients
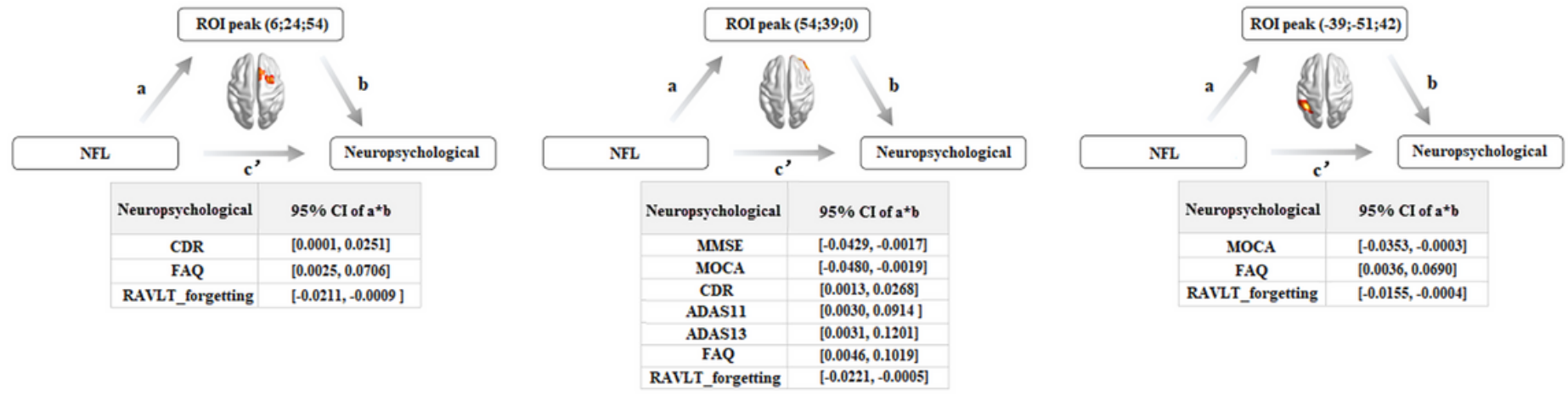

RAVLT_forgetting $\quad[-0.0221,-0.0005]$

\section{Figure 5}

Relationships between plasma NFL, functional connections, and cognition in AD-spectrum patients. (i) At baseline: there was a strong effect of plasma NFL on general cognitionand episodic memory mediated by the functional role of rightsuperior frontal gyrus (MNI: 6,24, 54), rightmiddle/inferior frontal gyrus (MNI: $5439,0)$ and right inferior frontal gyrus/thalamus (MNI: $3,-21,6)$, respectively $(p<0.05)$. (ii) At follow-up: three priori ROls included rightsuperior frontal gyrus (MNI: 6,24, 54), rightmiddle/inferior frontal gyrus (MNI: 5439, 0) and leftinferior parietal lobule/supramarginal gyrus (MNI: $-39,-51,42)$ as mediators, and plasmaNFL affected general cognitionand episodic memory by these mediators $(p<0.05)$. 